\title{
Environnements et sociétés dans les basses montagnes du Rif (Maroc) : des potentialités peu exploitées ${ }^{\mathrm{a}}$
}

\author{
T. Ftaïta ${ }^{1}$, H. Daïde ${ }^{2}$, M. Chaouki² ${ }^{2}$ A. Tribak ${ }^{2}$, A. Taous² et S. Perez ${ }^{3}$ \\ ${ }^{1}$ Laboratoire Interdisciplinaire Récits Cultures et Sociétés (LIRCES/EA3159), Université de Nice Sophia-Antipolis, \\ 98 boulevard Edouard Herriot, 06204 Nice, France \\ 2 Laboratoire d'analyses géoenvironnementales et d'aménagement (LAGEA), Université Sidi Mohamed Ben \\ Abdellah de Fès, Route d'Imouzzer, BP. 59, Fès, Maroc \\ ${ }^{3}$ UMR 7300 ESPACE, Université de Nice Sophia-Antipolis, 98 boulevard Edouard Herriot, 06204 Nice, France
}

\begin{abstract}
Résumé. L'agriculture marocaine représente seulement $19 \%$ du PIB national alors que $40 \%$ de la population vit de ce secteur, elle connait aujourd'hui de profondes transformations sous l'impulsion d'un Plan national «Maroc Vert» (PMV) qui vise à améliorer la productivité agricole, et par là même contribuer à un meilleur revenu pour les agriculteurs (fellahs). Le Rif qui a longtemps été un espace en marge des centres décisionnels n'échappe pas à cette volonté. Malgré des contraintes que nous aborderons, des potentialités agricoles et touristiques existent qui valoriseraient cet espace, et ce, dans un contexte de développement durable. Nous proposons dans cet article de faire un premier point sur les valeurs ajoutées envisageables, ainsi que sur une nouvelle méthodologie employée pour les évaluer à savoir l'outil Bayesia Expert Knowledge Elicitation Environment(BEKEE). Des exemples d'espaces déjà mis en valeur, notamment par des techniques d'irrigation modernes sont également présentés.
\end{abstract}

\section{La région du Sud Rifain au Maroc, des ressources riches et diversifiées mais peu valorisées}

Constituée de basses montagnes, la région du Sud rifain, située au nord de Fès (Figure 1), jouit de potentialités naturelles variées. Toutefois, la population n'en bénéficie que d'une manière limitée.

\subsection{Une densité humaine très forte}

La région se caractérise par un surpeuplement humain apparent. La densité humaine rurale moyenne atteint $100 \mathrm{hab} / \mathrm{km}^{2}$. Elle culmine même à $260 \mathrm{~km}^{2}$ dans la commune rurale de Bouadel. C'est une densité très importante, eu égard à l'exiguïté des terres agricoles, ne dépassant guère 5 ha par exploitation, au caractère accidenté du relief, et à l'intensité de la dégradation spécifique (1 000 et 3600 tonnes $/ \mathrm{km}^{2}$ ).

\subsection{Ressources agricoles et produits du terroir :}

L'ancienneté de la sédentarisation de la population a fortement façonné le paysage agraire des montagnes (Figure 2). L'extension spatiale de l'oliveraie en témoigne, le nombre moyen d'oliviers pour les exploitations de moins de 5 ha, aussi majoritaires qu'elles soient, étant de 40 pieds

\footnotetext{
${ }^{a}$ Cet article s'inscrit dans le cadre d'une recherche financée par le programme PHC-Volubilis.
}

à l'hectare. Néanmoins, le rendement reste faible, seulement 14L/Q1.

Le figuier est également très présent. Les figues de la région, dites «figues de Mtioua », sont tellement appréciées que leur commercialisation dans les villes régionales et nationales se fait commodément. La région est connue aussi pour ses céréales biologiques, peu d'agriculteurs utilisant les engrais, les pesticides chimiques ou les semences hybrides. Toutefois, l'intégration du cannabis dans les exploitations agricoles laisse peu de place à l'assolement céréalier. Les paysans justifient cette nouvelle orientation par le fait que les gains issus d'un hectare de cannabis peuvent atteindre 80000 dirhams par an, alors que ceux d'un hectare de blé tendre, étant d'ailleurs instables, ne peuvent dépasser 2500 dirhams pendant une année pluviométrique normale (500 mm).

\subsection{Une couverture végétale riche mais en dégradation progressive}

La chênaie s'étend (chêne vert, chêne-liège, chêne tauzin, chêne zen) sur plus de 19100 hectares. Le tauzin et le zen couvrent 1300 hectares. S'ajoutent à cette formation plus de 4200 types de plantes aromatiques et médicinales dans le matorral (11300 hectares). Dans la région de Ghafsay, l'intégration du cannabis dans les exploitations agricoles prend une ampleur spectaculaire depuis le début des années quatre vingt dix. Toutefois, si les revenus tirés de cette culture améliorent les conditions de vie de la population rurale, ses effets dévastateurs sur la forêt et sur 


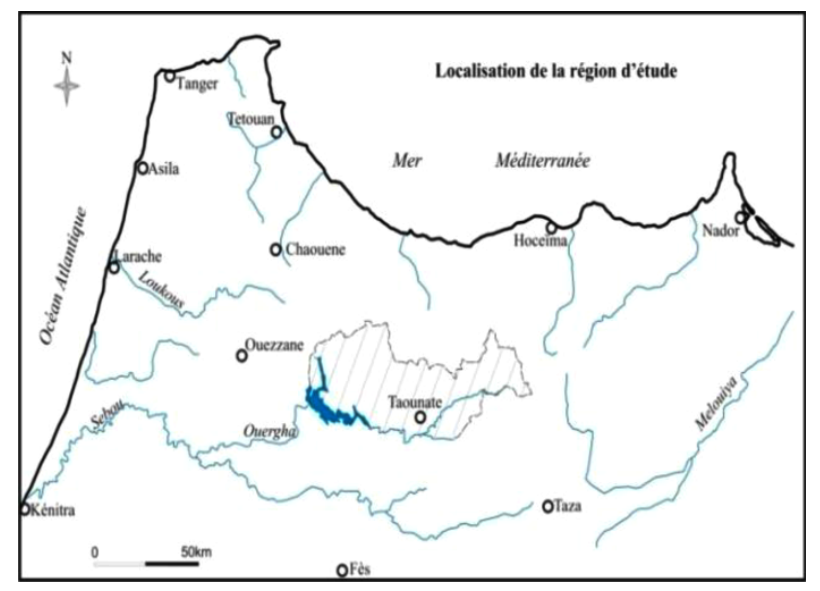

Fig. 1. La région du Sud rifain.

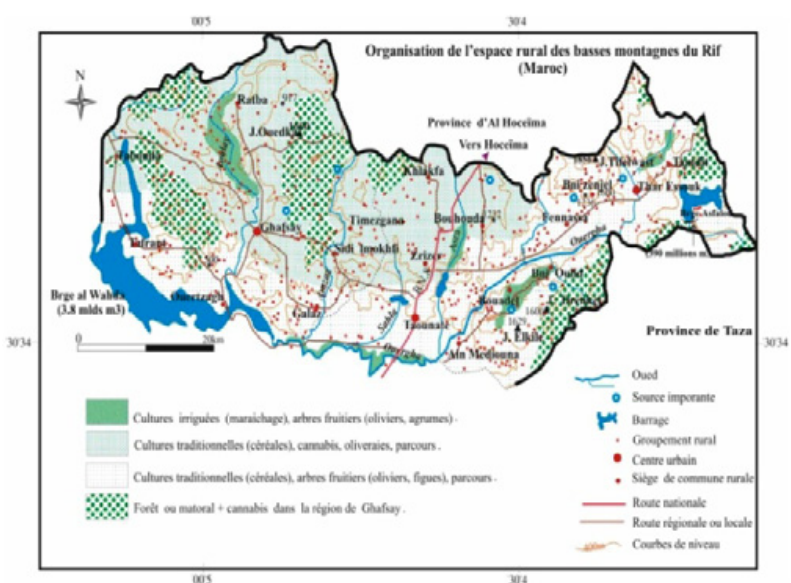

Fig. 2. Organisation de l'espace rural des basses montagnes du Rif.

la société ne cessent de se développer. Entre 1998 et 2007, le nombre d'infractions relatives au défrichement de la forêt a beaucoup augmenté à l'échelle des communes rurales de Ghafsay : + 243 infractions.

\subsection{Des potentialités touristiques très peu développées}

Les basses montagnes au sud du Rif central englobent de fortes potentialités éco-touristiques avec un riche patrimoine culturel et historique, des paysages agraires, une architecture rurale authentique, des monuments historiques, des souks ruraux et des Moussems. L'importance des précipitations pluviométriques (700 mm par an en moyenne) dote la région d'un paysage favorable au développement de l'écotourisme. Il s'agit essentiellemernt :

- de sources importantes telles la source de Bouadel dont le débit moyen annuel atteint $278 \mathrm{1} / \mathrm{s}$;

- d'oueds, à forts débits, celui de Ouergha étant d'environ $82 \mathrm{~m}^{3} / \mathrm{s}$ par an en moyenne ;

- de lacs artificiels à capacité élevée, le barrage d'Alwahda $\left(3,8\right.$ milliards de $\left.\mathrm{m}^{3}\right)$ étant à ce propos caractéristique ;

- d'une couverture forestière à espèces diversifiées (chêne zen, chêne tauzin...);
- de routes pittoresques classées routes internationales attrayantes, telles la route reliant Aïn Mediouna (à l'est de Taounate) et Thar Essouk.

Paradoxalement à ces potentialités éco-touristiques, la dynamique touristique est une des plus faibles à l'échelle nationale. Cet espace se trouve en marge des flux touristiques nationaux et régionaux.

\section{Une richesse naturelle mais des conditions de vie difficile}

\subsection{Faibles revenus agricoles et nécessité de recours à d'autres ressources économiques}

Les revenus agricoles se caractérisent par leur faiblesse et leur instabilité. Le revenu moyen annuel à l'hectare agricole, est de l'ordre de seulement 2700 dirhams. L'exiguité des terres agricoles et leur morcellement, le caractère accidenté du relief, l'intensité de la dégradation spécifique (1 000 et 3600 tonnes $/ \mathrm{km}^{2}$ ) sont autant de facteurs expliquant la contribution limitée de l'agriculture au budget familial. Par conséquent, le travail non agricole touche tous les ménages ruraux : dans la région ouest, aux alentours de la ville de Ghafsay, la couture (Casablanca, Fès), l'armée attirent une à deux personnes par ménages, alors qu'à l'ouest de la ville de Taounate l'émigration vers la France et les petits métiers urbains attirent eux aussi une population non négligeable.

\subsection{Une région fortement enclavée}

La densité globale des routes goudronnées dépasse de peu deux kilomètres pour 1000 habitants ruraux. Pendant l'hiver, l'anfractuosité du relief, la faible infrastructure routière et l'instabilité des sols (glissements fréquents) aggravent l'enclavement de la population et désavantagent la commercialisation des récoltes agricoles (olives) dans les souks hebdomadaires locaux et dans les villes avoisinantes (Fès).

Malgré ce diagnostic ces espaces sont riches de potentialités, mais comment les évaluer ? C'est ce que nous allons voir à présent.

\section{L'évaluation des potentialités de ces espaces}

La potentialité d'un espace peut être considérée comme la comparaison entre les éléments physiques qui le composent et qui relèvent du milieu, au sens large de la géographie physique (altitude, climat, conditions édaphiques, présence de l'eau...) avec les besoins de la population qui vit dans cet espace. Une potentialité surévaluée aboutit à une surexploitation des ressources. Une potentialité sous évaluée peut être socialement acceptable si les besoins de la population sont satisfaits, ou bien insatisfaisante dans le cas de poches de pauvreté. Lorsque les deux (ressources/besoins) sont en équilibre on parle d'optimum : les ressources ne subissent pas plus de pression anthropique qu'elles ne sont capables d'en absorber, elles sont alors adaptées au nombre d'habitants, et leur assurent un niveau de vie satisfaisant qui leur permet de couvrir l'ensemble de leurs besoins (nourriture, hébergement, accès à l'éducation des enfants etc...). 


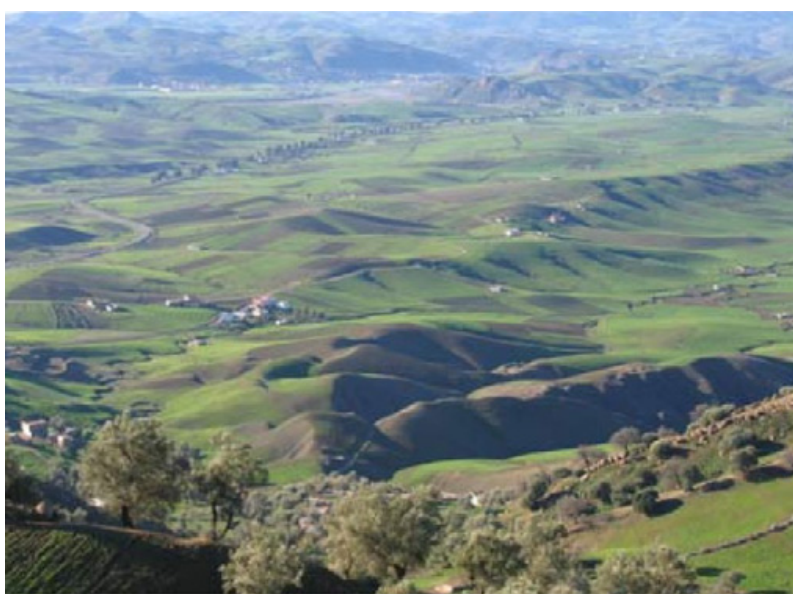

Fig. 3. Paysage collinaire dans la région de Taounate.

\subsection{Les indicateurs de potentialité retenus}

A partir d'une étude de l'occupation du sol par le biais de photographies aériennes nous envisageons d'attribuer un potentiel spatial à nos zones d'étude en fonction des critères suivants :

- \% de prairies naturelles et permanentes, de landes arbustives qui traduiraient les possibilités de pâturages pour l'élevage et qui viendraient en complément des autres productions agricoles, tout comme le $\%$ de couverture forestière, mais avec une gestion durable de la ressource en bois (exploitation/replantation),

- possibilités d'extension/réhabilitation de l'olivier, de l'amandier, du figuier, localisation de l'altitude limite pour l'olivier,

- présence de produits du terroir susceptibles d'être valorisés (figues, miel), plantes aromatiques et médicinales,

- possibilités de cultures de rente en substitution au cannabis : safran, rosacées, cultures maraîchères, vergers, betterave sucrière,

- \% de terres arables hors périmètre d'irrigation qui pourraient être valorisées par une irrigation de type goutte à goutte,

- ressource en eau disponible et en quantité suffisante,

- accessibilité des terrains par rapport aux douars (villages),

- pente des terrains,

- possibilités de remembrement.

En ce qui concerne le potentiel touristique nous retiendrons comme critères l'existence de plans d'eau, de points de vue remarquables, la valeur esthétique des paysages, la possibilité d'offres d'hébergement, l'accessibilité, et là encore une ressource en eau pérenne. A noter qu'il y a dans cette succession de paysages collinaires un potentiel pour un tourisme qui réponde aux besoins de repos, de calme et de tranquillité de métropolitains stressés (Figure 3).

\begin{tabular}{|c|c|c|c|}
\hline Région administrative & $\begin{array}{l}\text { Vulnérabilité } \\
\text { au CC (\%) }\end{array}$ & $\begin{array}{l}\text { Potentiel } \\
\text { agricole (mm) }\end{array}$ & Classement \\
\hline Chaouia - Ouardigha & 80 & 422 & 1 \\
\hline Grand Casablanca & 79 & 408 & 2 \\
\hline Rabat - Salé - Zemmour - Zaër & 37 & 524 & 3 \\
\hline Tadla-Azilal & 33 & 523 & 4 \\
\hline Doukkala - Abda & 51 & 334 & 5 \\
\hline Gharb - Chrarda - Beni Hssen & 23 & 607 & 6 \\
\hline Marrakech - Tensift - Al Haouz & 32 & 352 & 7 \\
\hline Fès - Boulemane & 16 & 358 & 8 \\
\hline Oriental & 14 & 275 & 9 \\
\hline Meknès - Tafilalet & 12 & 313 & 10 \\
\hline Tanger - Tetouan & 4 & 814 & 11 \\
\hline Taza - Al Hoceima - Taounate & 3 & 489 & 12 \\
\hline Sous - Massa - Drâa & 3 & 254 & 13 \\
\hline Guelmim - Es Semara & 0 & 76 & 14 \\
\hline LaÂyoune - Boujdour - Sakia El Hamra & 0 & 38 & 15 \\
\hline Oued Ed Dahab - Lagouira & 0 & 39 & 16 \\
\hline
\end{tabular}

Fig. 4. Vulnérabilité au changement climatique des régions marocaines. Source : Rapport de faisabilité du Projet d'Intégration du Changement Climatique dans la Mise en oeuvre du Plan Maroc Vert (PICCPMV).

L'objectif est d'identifier grâce à ces indicateurs :

1) des espaces sans contraintes majeures susceptibles de supporter un large spectre de productions,

2) des espaces à faibles contraintes, facilement corrigeables sans impacts économiques ou environnementaux majeurs,

3) des espaces à fortes contraintes, aménageables mais nécessitant des interventions lourdes ou inadaptées par exemple pour une mise en culture.

Le tout dans une perspective de développement durable, en fonction des contraintes actuelles, mais aussi futures (manque d'eau, accroissement démographique), quoique la région du Rif (Taza-Al Hoceima-Taounate) ne soit pas a priori vulnérable au changement climatique comme nous pouvons le voir sur la Figure 4.

L'analyse des photographies aériennes nous permettra de quantifier pour chaque espace les indicateurs de potentiels retenus. Les résultats seront bien entendu validés sur le terrain, mais aussi par la connaissance des différents experts locaux. L'originalité de notre démarche c'est que désormais cette connaissance fera l'objet d'une quantification grâce aux réseaux bayésiens et à un nouvel outil développé dans le logiciel BayésiaLab : BEKEE (Bayesia Expert Knowledge Elicatation Environment) [1].

\subsection{Présentation des réseaux bayésiens et de l'outil BEKEE}

La formalisation de la théorie des réseaux bayésiens est issue des travaux de Judea Pearl (chercheur à l'UCLA) et Finn Jensen (Chercheur à l'Université d'Aalborg au Danemark) dans les années 80. La naissance des réseaux bayésiens vient du fait que ces chercheurs souhaitaient intégrer la notion d'incertitude dans les systèmes experts. Les réseaux bayésiens peuvent s'apparenter à un graphe dont les nœuds définissent les variables du système et dont les arcs définissent l'existence de relations entre ces variables. Ils décrivent de manière qualitative des relations de dépendances ou d'indépendances conditionnelles entre les variables et quantifient ces mêmes relations par le biais de probabilités conditionnelles.

Un réseau bayésien est donc un graphe causal, auquel s'ajoutent des éléments de probabilités issus de la théorie 
bayésienne. Les relations causales ne sont pas absolues, mais relatives, elles sont en fait associées à une probabilité indiquant le degré de croyance que l'on a dans l'évènement, tel que par exemple le degré de croyance que l'on a dans le fait qu'un espace peut potentiellement évoluer vers une autre occupation du sol plus rentable pour la population et sans contraintes environnementales majeures.

Les réseaux bayésiens possèdent en outre, un caractère intéressant en géoprospective puisqu'ils permettent de prendre en compte la notion d'incertitude. Incertitude liée au fait que si l'expert estime le potentiel d'un espace, pour que ce potentiel se réalise, celui-ci doit rencontrer l'aval des acteurs locaux (notables, chefs de famille).

Il est également possible de modéliser des variables correspondant à des politiques d'aménagements à mettre en place ou bien à des décisions à prendre pour remédier à un problème. Ces variables peuvent être hiérarchisées en donnant un poids à chaque interaction qui peut représenter un coût ou un bénéfice. Ainsi, les réseaux bayésiens peuvent avoir un caractère décisionnel en aidant à choisir une solution plutôt qu'une autre en fonction du poids de son interaction. Ces réseaux permettent de calculer des probabilités conditionnelles d'événements reliés les uns aux autres par des relations de cause à effet. Cette utilisation s'appelle l'inférence. La mise en place du réseau se fait en associant au formalisme graphique (structure du réseau), un contenu probabiliste (paramètres du réseau).

Les variables retenues peuvent être aussi bien quantitatives que qualitatives, et nous allons nous attacher à analyser leurs interrelations, leurs combinaisons, et quantifier ces relations à l'aide de paramètres ou de probabilités. Le contexte est celui d'un formalisme mathématique de représentation des connaissances incertaines (probabilistes) particulièrement bien adaptées aux potentialités spatiales.

De plus, l'outil de type «réseau bayésien» a l'avantage de pouvoir rassembler et fusionner des connaissances à partir de données de diverses natures quantitatives/qualitatives, de types expert, de les faire cohabiter dans un même modèle, mais également d'introduire le retour d'expériences (données historiques ou empiriques). En effet, un réseau bayésien peut être construit à partir de données, soit par apprentissage (apprentissage incrémental, inférence), soit à partir d'une modélisation explicite du domaine de type expert, soit il permet de coupler les connaissances des experts et les données disponibles. Les données peuvent être exprimées sous forme de règles logiques, de type équations statistiques ou bien encore de probabilités subjectives, voire d'observations.

Grâce à la fusion de données, le réseau bayésien a la capacité de prendre en compte des données incomplètes ou incertaines et de vérifier ensuite ces informations. En effet, connaissant les valeurs d'une variable mais méconnaissant les valeurs d'autres, les données manquantes ou lacunaires peuvent être retrouvées grâce au calcul des probabilités (principe là encore de l'inférence). Enfin, par le biais de procédures de type Data mining les meilleures variables à sélectionner sont proposées, de même que des informations a priori inconnues et à valeurs prédictives peuvent être «automatiquement» extraites de la base de données. En effet, les réseaux bayésiens permettent à la fois de modéliser des connaissances et à la fois de produire des connaissances (fonction hautement heuristique) à travers la mise en évidence de relations causales jusqu'à présent cachées ou de variables latentes, et ce, dans le cadre d'une analyse non supervisée (Causal knowledge discovery).

Les réseaux bayésiens apportent donc de la valeur ajoutée en termes de connaissances, et de potentialités spatiales, ils représentent un formidable outil pour la modélisation quantitative de systèmes complexes en domaine incertain (potentiel spatial).

Avec l'outil BEKEE nous allons traduire la connaissance tacite des experts, en connaissance explicite (Elicitation) et quantifiable sous forme de probabilités. Les portions d'espaces étudiées seront représentées par des nœuds du réseau, ces nœuds seront reliés à d'autres variables qui correspondront aux indicateurs précédemment retenus. Chaque expert attribuera au regard de ces critères et de sa connaissance du terrain un poids, une probabilité d'émergence du potentiel (agricole et/ou touristique) pour chaque espace d'étude, tandis que les arcs du modèle définiront les relations probabilistes entre les différentes variables. Sachant que les potentiels des parcelles sont conditionnés par : l'état des indicateurs, la connaissance des experts, mais également par l'état des autres variables, il devient alors possible de simuler les effets d'une décision (plus d'irrigation), ou l'évolution d'une variable (plus de population) à la fois sur toutes les variables et sur toutes les parcelles...

\section{Accès et gestion de l'eau et des ressources naturelles : vers une valorisation des savoirs et savoir-faire des communautés rurales locales}

La gestion des ressources naturelles dans les zones rurales du Maroc a longtemps été marquée par le poids des institutions traditionnelles nommées localement Djemaa (assemblée représentative en charge des affaires économiques et politiques). Ces institutions communautaires disposaient d'un arsenal juridique consigné dans des droits coutumiers locaux, oraux ou écrits [2]. Chaque droit coutumier est à l'image de la communauté qui l'a produit dans le sens qu'on ne peut l'étudier et en cerner sa portée sans le replacer dans son contexte écologique, historique et politique. Force est de constater que là où ces coutumiers ont perdu de leur légitimité l'autorité de la Djemaa s'est effritée, cela correspond aujourd'hui à des espaces où les équilibres Société/Environnement sont les plus menacés. Par le passé, ces communautés rurales veillaient à maintenir un équilibre entre Ressources et Prélèvements conditionnant ainsi les modalités d'accès et d'appropriation des ressources naturelles dans un souci de prévenir les conflits d'usages et de sanctionner le cas échéant les dérives [3].

Ce volet nous paraît fondamental pour asseoir de manière endogène les actions de développement et maintenir une cohésion sociale des communautés en présence. Il s'agira également, dans cette partie de notre recherche, de valoriser les savoirs et savoir-faire locaux et les mettre à contribution dans le cadre d'une nouvelle gouvernance des ressources naturelles seule capable, à nos yeux, d'assurer une réelle durabilité. Cette partie de la recherche s'intéressera en priorité à la gestion des ressources en eau dans le nouveau contexte institutionnel relatif à la gestion de l'eau au Maroc : loi relative à la mise en place des 


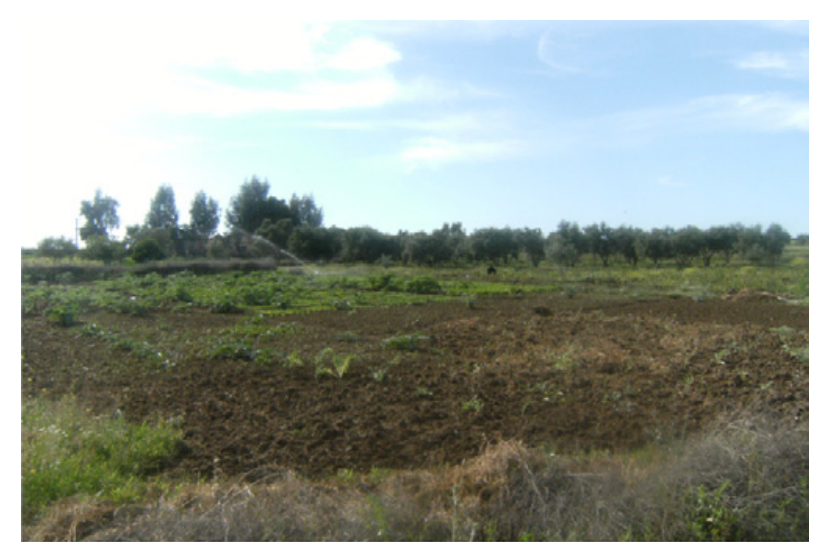

Fig. 5. Parcelle irriguée par aspersion - Périmètre Sahla (C) T. Ftaïta).

associations des usagers des eaux agricoles en comparaison avec le modèle de la gestion communautaire locale de l'eau (l'eau peut être appréhendée comme patrimoine matériel et immatériel). La gestion des ressources en eau ne se limite pas à ses seuls aspects techniques, mais elle doit prendre en considération les aspects sociaux et politiques de l'appropriation et de la distribution ; aspects souvent ignorés par les techniciens et les aménageurs, d'où les nombreux conflits d'usages et d'appropriations. En effet, la question de l'eau est et demeure une question éminemment sociale et politique.

Deux terrains de recherche ont été identifiés dans cette étude portant sur la gestion sociale de l'eau. La première concerne la gestion traditionnelle et communautaire de l'eau dans la localité de Bouadel où l'eau est appropriée collectivement et où l'irrigation est ancestrale. L'eau, dans ce type de communauté, s'avère être l'élément structurant des communautés locales. Le deuxième terrain s'intéresse au projet d'irrigation moderne dit projet Sahla. Ce projet Sahla tire sa ressource en eau du barrage éponyme. Ce dernier a été édifié pour assurer l'alimentation en eau potable de la petite ville de Taounate et pour la mise en valeur des terres Bours par l'irrigation. Le projet Sahla visant l'irrigation des anciennes terres cultivées en sec a été financé par l'UE et le Maroc pour la mise en irrigation d'une superficie de 2794 ha. La nouveauté de ce projet est à la fois technique et institutionnelle. En effet, l'irrigation s'inscrit dans la modernisation des techniques d'irrigation en adoptant soit la technique du goutte à goutte, ou de l'aspersion. Toute irrigation gravitaire est strictement prohibée sous peine de sanction administrative. Les deux orientations techniques visent une économie de l'eau par une gestion rationnelle des ressources disponibles et le développement des cultures rentables.

En effet, nous observons sur le terrain que certains agriculteurs se sont orientés vers des cultures à haute valeur économique ajoutée et ont introduit la culture du safran, culture jusque là étrangère à la région, car elle est historiquement développée dans le sud du Maroc. D'autres se sont orientés vers la culture des plantes aromatiques et médicinales. Cette dernière orientation a été largement facilitée par la proximité du périmètre de l'Institut National des Plantes Médicinales et Aromatiques (INPMA Taounate) : un Institut qui dispose de toute l'expertise

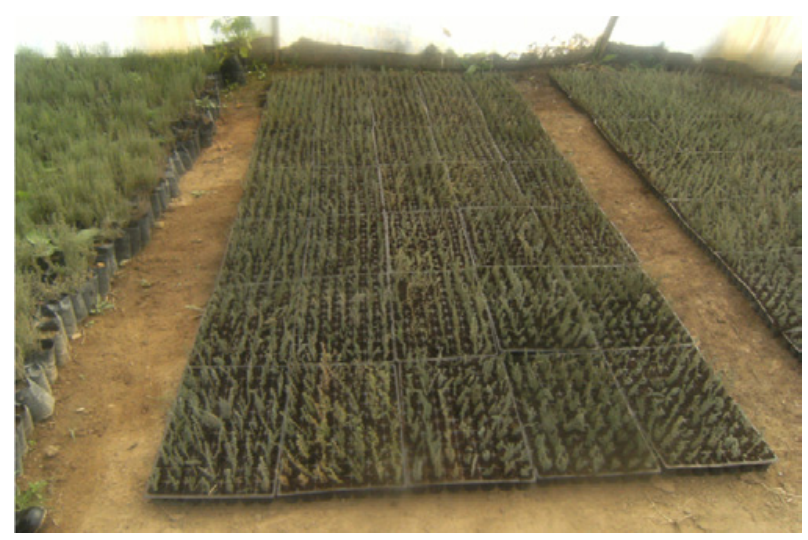

Fig. 6. Pépinières pour les plantes aromatiques et pour la vente des bulbes de safran aux agriculteurs - Périmètre irrigué de Sahla, novembre 2011 (C) T. Ftaïta).

scientifique et technique nécessaire à l'extraction d'huiles essentielles $^{\mathrm{a}}$ et qui est en mesure d'aider les agriculteurs locaux. L'Institut dispose d'une grande superficie où l'on cultive de nombreuses plantes. Les techniciens et les ingénieurs procèdent à toutes les phases visant ainsi la distillation des plantes suivant des procédés scientifiques répondant aux normes internationales. Il nous semble important que cet Institut puisse prendre part aux différents projets des agriculteurs désireux d'investir dans ce type de production.

La deuxième innovation est d'ordre institutionnel, elle consiste en l'adoption du nouveau principe de la gestion participative des irrigants en se constituant en Associations des Usagers des Eaux Agricoles conformément à la nouvelle loi de 1992. Dans un cadre associatif, on ne peut parler d'ayants droit à l'eau, comme dans le système traditionnel où l'agriculteur dispose d'un droit d'eau coutumier (appropriation communautaire collective), mais simplement d'usagers des eaux agricoles : l'Etat demeure le propriétaire des ressources en eau et des aménagements hydrauliques). Par ailleurs, les bénéficiaires de l'eau d'irrigation constitués en association devront, dans le cadre de ces AUEAs, s'acquitter d'une cotisation et d'une redevance liées à la consommation de l'eau pour l'irrigation. La gestion du périmètre d'irrigation, contrairement au modèle traditionnel de type Bouadel, est confiée à ces AUEAs.

Le projet Sahla compte sept AUEAs et une Fédération regroupant l'ensemble de ces AUEAs. Les sept AUEAs sont divisées en deux entités administratives : quatre AUEAs relevant d'une commune rurale et les trois autres de la commune urbaine de la petite ville de Taounate (Cf. Carte des AUEAs du projet Sahla).

Le projet Sahla, est un projet novateur dans le sens où les pouvoirs publics et les administrations de l'Etat (autorités locales, Direction Provinciale de l'Agriculture, Agence de Bassin) et le bailleur de fonds ont souhaité intégrer dès le départ du projet, suivant ainsi les principes d'une gestion participative des irrigants largement colorée

\footnotetext{
${ }^{a}$ Nous tenons à remercier notre collègue Abdessalam Khanchoufi Directeur de INPMA Taounate ainsi que toute son équipe pour leur accueil et leur disponibilité.
} 


\begin{tabular}{|l|c|c|}
\hline Nom de l'AUEA & $\begin{array}{c}\text { Nombre } \\
\text { adhérents }\end{array}$ & $\begin{array}{c}\text { Superficie } \\
\text { dominée (ha) }\end{array}$ \\
\hline AL & 426 & 237 \\
MOSTAKBAL & 472 & 461 \\
RAHMA & 258 & 560 \\
AL FALAH & 345 & 188 \\
CHARAF & 188 & 499 \\
NACER & 295 & 610 \\
AL IZDIHAR & 127 & 239 \\
TADAMOUN & & \\
\hline & & $\mathbf{2 7 9 4}$ \\
\hline
\end{tabular}

Fig. 7. Source : Organe de Gestion du périmètre Sahla (OGP), mai 2012.

par le «local» et le «communautaire », les futurs usagers de l'eau par la mise en place d'une équipe d'animateurs à la disposition des agriculteurs. Cette tentative de participation des futurs usagers du périmètre irrigué est une innovation en soi, car très souvent les usagers ne sont sollicités et mobilisés qu'après achèvement du projet d'irrigation [4] Or, malgré ces initiatives, la mobilisation des futurs usagers de l'eau est loin d'être salvatrice. Très peu d'agriculteurs ont réellement investi dans la mise en irrigation de leurs parcelles (nombreuses sont encore les parcelles cultivées en sec). Les raisons sont multiples. Certaines relèvent des contraintes liées au remembrement des terres : une phase souvent complexe et qui oppose les propriétaires terriens au service du remembrement. Plusieurs litiges nous ont d'ailleurs été signalés. L'autre contrainte qui n'est pas la moindre réside dans le caractère juridique de certaines parcelles. En effet, l'indivision de l'héritage créé des difficultés supplémentaires : quel héritier est en mesure de rependre légitimement l'exploitation devant l'administration ? Enfin, notons l'ignorance des exigences de l'agriculture irriguée moderne (n'oublions pas que les parcelles formant le nouveau périmètre irrigué du Sahla étaient cultivées en $s e c$ et que les agriculteurs ne disposent pas de savoirs et de savoir-faire traditionnel en matière d'irrigation). Tous ces aspects rendent difficile toute innovation en retardant l'équipement technique des parcelles (achat de matériel de goutte à goutte ou des asperseurs) et par conséquent la mise en irrigation effective ${ }^{\mathrm{b}}$.

C'est dans un souci d'efficacité et de facilitation technique que la gestion du périmètre irrigué a été confiée dans un premier temps à un organe de gestion du périmètre Sahla (OGP), constitué par une équipe d'ingénieurs et de techniciens en irrigation. Cet organe devra confier la gestion effective du périmètre à la fédération des AUEAs qui en prendra la relève. Durant la phase transitoire (2012-2014), l'administration assurera les frais de gestion, à charge pour les AUEAs / Fédération de prendre la relève en payant, par la suite, les salaires des techniciens de l'organe de gestion du périmètre irrigué Sahla, quant à la maintenance des infrastructures lourdes, elle sera confiée à une entreprise privée spécialisée.

Il est clair que la nature du projet, ses exigences techniques et financières nécessitent une organisation

\footnotetext{
${ }^{\mathrm{b}}$ Nous remercions notre collègue Kamal Belabbes, Expert en irrigation en charge du projet Sahla, et toute son équipe, pour leur disponibilité et pour les échanges constructifs que nous avons eus.
}

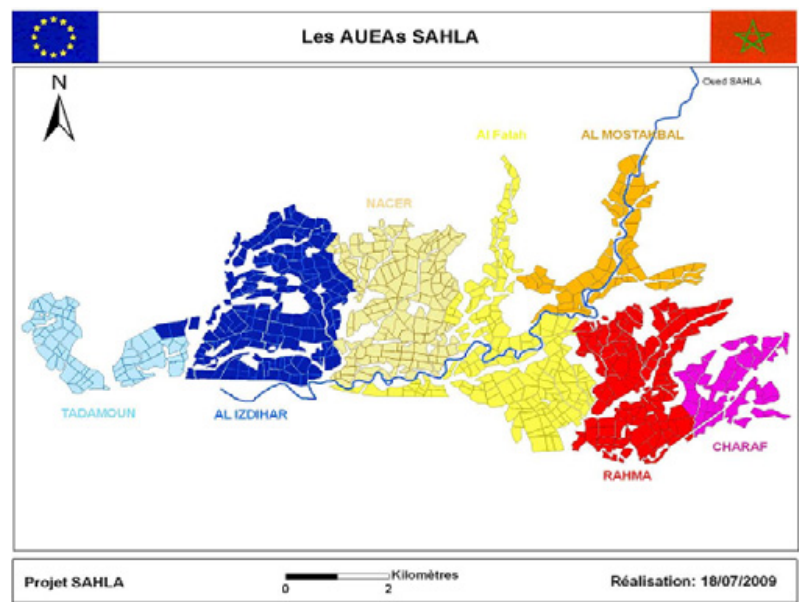

Fig. 8. Source : Organe de Gestion du périmètre Sahla (OGP), mai 2012.

sociale et économique sans faille. Tous les pouvoirs sont dans les mains des responsables des AUEAs et de la Fédération. Par ailleurs, il est un peu tôt pour en faire une lecture approfondie. La mise en eau effective du périmètre est récente : elle date de 2010. Les premières enquêtes de terrain auprès des membres de l'organe de gestion, des Présidents d'AUEAs et de la Fédération, ont révélé que peu d'agriculteurs ont équipé, à ce jour, leurs parcelles en goutte à goutte ou aspersion. Pourtant une aide financière conséquente de l'Etat couvre quasiment à $100 \%$ les frais engagés par l'agriculteur. A cela, s'ajoutent les aides de l'administration en matière de distribution gratuite de plants, notamment pour les arbres fruitiers. Ce qui ressort, de nos premières enquêtes c'est que la greffe s'avère difficile auprès d'agriculteurs qui par ailleurs ignorent les techniques d'irrigation traditionnelles et qui par conséquent ne peuvent se projeter dans une agriculture moderne irriguée. Pourtant, les potentialités existent, mais sans aucune transmission des savoirs et savoir-faire, il devient difficile d'inscrire des groupes sociaux avec des logiques et des stratégies différentes. Les enjeux du pouvoir entre la commune rurale en charge de 4 AUEAs et la commune urbaine de Taounate qui en contrôle 3 ne facilite pas la tâche, d'où le retard en matière d'équipement des parcelles, et par conséquent de leur mise en culture.

Par opposition, le modèle de la gestion de l'eau et de l'irrigation dans la localité de Bouadel et des villages appartenant à la même commune rurale s'avère radicalement différent. Cette différence n'est pas seulement de nature technologique, mais elle trouve son essence dans le mode d'organisation sociale traditionnelle des communautés en présence.

L'eau est disponible, les sources de Bouadel sont très importantes : plus de 280 litres par seconde. Cette eau est rigoureusement partagée entre les différents villages. Un partage et une partition de l'eau qui trouve son explication dans l'histoire sociale et politique de la région. Les modalités de distribution de l'eau entre les ayants droit (ici les agriculteurs disposent d'un droit d'eau et ne peuvent être considérés comme de simples usagers comme c'est le cas dans le périmètre irrigué de Sahla) diffèrent d'une localité à une autre suivant les coutumes locales. Les usages de l'eau sont aujourd'hui diversifiés. Si par le passé, l'eau 


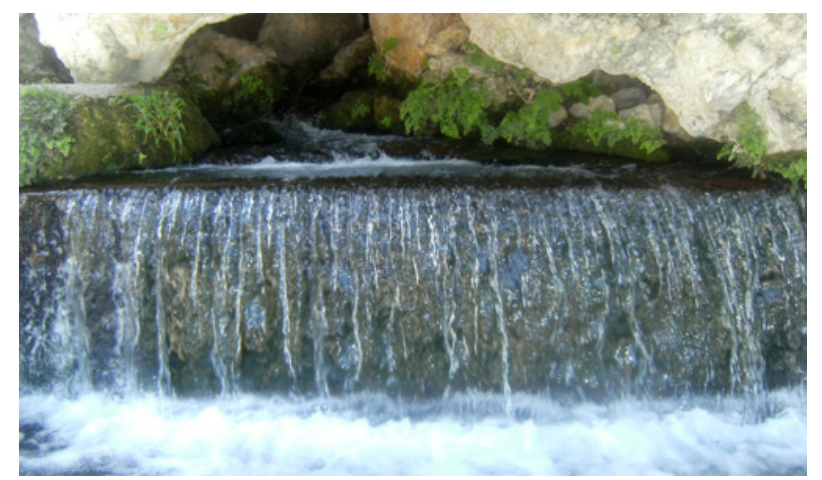

Fig. 9. Source du village de Bouadel (C) T. Ftaïta).

servait avant tout à l'irrigation et aux usages domestiques, aujourd'hui la pression sur l'eau est de plus en plus grande. Certes, le secteur agricole irrigué demeure le plus important, mais de plus en plus les demandes en eau potable se font sentir eu égard à l'accroissement démographique et à l'absence d'une desserte en eau potable moderne des ménages (actuellement le recours à des camions citernes pour alimenter certains villages s'avère nécessaire pour répondre à ces besoins). Un autre usage a vu le jour ces dernières années, il s'agit des activités touristiques notamment dans le village de Bouadel où se trouvent les sources portant le même nom. Désormais, une piscine a été construite au cœur de la vallée dénaturant à jamais un site naturel. Si ces activités touristiques sont importantes pour améliorer les conditions de vie d'une frange de la population, la mise en pace d'activités touristiques sans tenir compte de leurs conséquences, à la fois sur le plan écologique/paysager et socio-économiques, conduisent à engendrer des effets pervers qu'il conviendra de rectifier. La présence de cette piscine, sur un site traditionnellement fréquenté par les familles, dans un pays et une localité où les traditions sont encore vivaces, et où le respect de l'autre passe par le respect de soi-même, pose un énorme problème. Certaines familles ne peuvent fréquenter le site car trop de jeunes et non jeunes sont dénudés ce qui pose un problème de cohabitation. Il est clair que le choix du site n'est pas optimal alors que sa mise en place à l'entrée du village ne poserait aucun problème. A cela, s'ajoute l'absence de structures d'accueil pour les visiteurs (pas d'hôtel et encore moins de gites familiaux). Les visites se font durant la journée créant ainsi une surpopulation la journée et un vide pendant la soirée. Or, là encore des potentialités existent.

La richesse en eau de la région de Bouadel a été l'objet d'une grande convoitise de l'ONEP (Office National de l'Eau Potable). A la fin des années 90, un projet, financé à $90 \%$ par l'AFD (Agence Française pour le Développement) ayant pour but l'alimentation en eau potable de la région de Bouadel a conduit à une virulente confrontation entre les habitants de la région et les autorités locales. Sans aucune concertation avec les communautés locales, le projet de l'ONEP a été présenté comme une réalisation en vue d'améliorer l'accès à l'eau potable. Or, ce projet était ressenti par la population locale comme une tentative de main mise sur les eaux de la région. Malgré l'avancée des travaux (les grandes infrastructures) entrepris par l'ONEP, la population locale s'est opposée farouchement à la poursuite du projet. Les tentatives d'intimidation des autorités locales

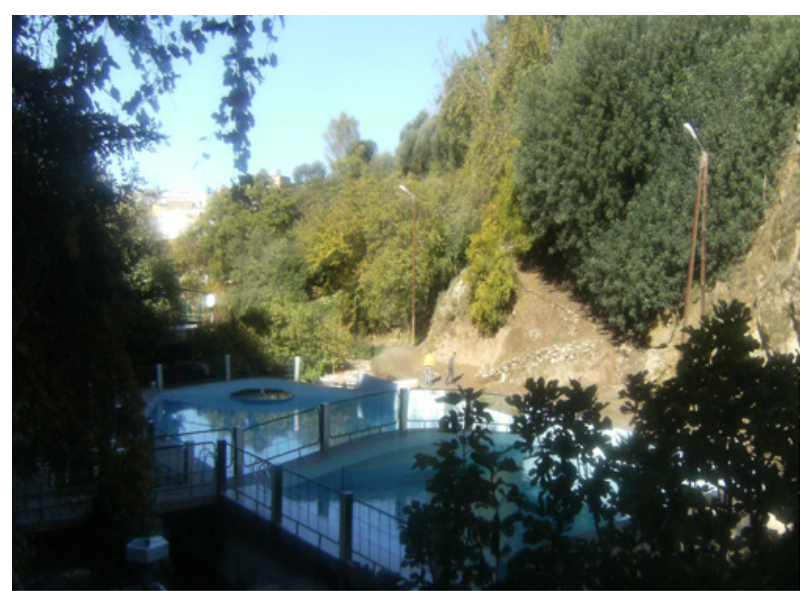

Fig. 10. Piscine de Bouadel au cœur du site des sources d'eau du village (C) T. Ftaïta).

n'ont eu aucun effet sur la cohésion et l'opposition des communautés locales. Les habitants ne souhaitant pas se déposséder de leur bien le plus cher, à savoir l'eau, ont tenu bon et le projet a été bel est bien abandonné grâce à la mobilisation des communautés locales. Cet incident montre si besoin est les enjeux actuels et la pression grandissante sur les ressources en eau de la région. La cohésion sociale est plus grande lorsqu'il s'agit d'une appropriation collective des ressources naturelles. En effet, l'appropriation des ressources en eau de la région est partagée collectivement par de nombreux villages de la zone. Et l'on ne peut intervenir dans aucun projet si l'on prend pas en considération l'histoire sociale et politique des communautés locales. Ce qui montre la complexité et la diversité des réalités locales dont il faut tenir compte dans toute analyse.

La région de Bouadel demeure, à plusieurs égards, une région très intéressante non seulement parce qu'elle offre un modèle ou plutôt des modèles de gestion équilibrés de l'eau et des ressources naturelles en général, mais parce que les activités agricoles et leur conduite nous enseignent sur un héritage technique et social largement façonné par le mode d'organisation locale. On ne peut prétendre ici que les enjeux de pouvoir et les conflits entre ayants droit à l'eau sont inexistants, cependant la gestion collective des ressources naturelles impose une solidarité communautaire que l'on ne retrouve pas dans les périmètres irrigués modernes (de typeSahla). Par ailleurs, l'héritage de ces savoirs et savoir-faire tant agronomique que technique et juridique doit être mobilisé et réinventé pour maintenir l'équilibre entre la société et son environnement, en d'autres termes entre ressources disponibles et prélèvements. A titre d'exemple, dans la région de Bouadel, il n'est pas rare de trouver dans un même carré de culture plus de 15 variétés ce qui atteste d'une biodiversité, alors que dans les périmètres modernes la monoculture est de rigueur.

Au-delà des aspects relatifs à la gestion des ressources naturelles, et aux apports des savoir-faire traditionnels qu'il convient de mobiliser et de valoriser pour une gestion durable de ces ressources, notre projet de recherche vise à travers de nombreux diagnostics, à améliorer les modes de productions locaux et les conditions de vie de la population locale. 


\subsection{L'amélioration des systèmes de production et la diversification des cultures}

Le diagnostic des modes locaux de productions nous permettra de mieux cerner et comprendre les chaînes opératoires et d'en souligner les apports et les limites. Sur le plan de la technologie agricole, l'utilisation de certaines techniques traditionnelles ne peut être appréhendée comme signe d'une agriculture archaïque, car là aussi certaines techniques modernes s'avèrent inopérantes en fonction du milieu naturel et de ses potentialités. Les recommandations tiendront compte des capacités du milieu naturel, des savoirs et savoir-faire locaux, ainsi que des capacités financières des agriculteurs à s'engager dans une modernisation des techniques agricoles, voire dans une nouvelle orientation agricole. Le développement ne veut nullement dire faire table rase des techniques traditionnelles.

\subsection{L'amélioration des conditions de vie et la diversification des sources de revenus des agriculteurs}

Nul doute que la diversification des revenus locaux est souvent fonction des potentialités des régions, de leurs situations en matière d'enclavement et leurs forces vives mobilisables (émigration, présence des jeunes). Cependant, les communautés agricoles locales ont souvent cherché à diversifier les produits agricoles se méfiant ainsi des méfaits de la monoculture. Or, les transformations actuelles que subissent les zones rurales tant au niveau institutionnel qu'au niveau anthropologique nous obligent à prendre avec précaution certaines visions « développementistes». Quant à l'amélioration des conditions de vie, il est clair qu'à l'heure de la mondialisation, les communautés rurales ne peuvent plus être appréhendées comme vivant dans un perpétuel présent ethnographique, elles aspirent à des conditions de vie aussi proches que celles dont disposent les populations urbaines. Il semble qu'un minimum d'infrastructures de base doit être envisagé pour répondre aux besoins élémentaires : santé et éducation. Nous savons que des efforts ont été consentis dans certaines régions rurales du Maroc notamment dans le domaine de l'accès à l'eau potable et à l'électrification. La mobilisation de la société civile notamment des ONGs nationales et internationales, les programmes bilatéraux internationaux de coopération ont contribué à l' amélioration de la situation. Qu'en est-il de notre région ? Comment, faire participer la population locale à l'élaboration des choix, ainsi qu'impliquer l'ensemble des acteurs y compris les institutionnels dans la prise en charge de ces besoins ? Autant de questions auxquelles cette recherche tentera de répondre.

\subsection{La mise en place d'activités génératrices de revenus, comme l'apiculture, l'artisanat, l'écotourisme et l'agriculture biologique}

Si les activités génératrices de revenus sont souvent avancées comme solutions aux problèmes du monde rural, dans la pratique, leur mise en place effective s'avère plus complexe. Quels revenus ? Pour quelle catégorie de population ? Autant de questions et d'enjeux qui dépassent le cadre des propositions et qui doit davantage s'attacher aux réalités locales, aux stratégies et logiques des acteurs sociaux. En réalité, les propositions et recommandations tiendront compte des interactions entre faits écologiques et faits anthropologiques et économiques. Ainsi, chaque activité doit répondre à des critères écologiques (potentialités, disponibilités, risques de surexploitation), à des critères techniques, à la mobilisation des savoirs et savoir-faire, ainsi qu'aux retombées économiques et à leur redistribution. Le développement économique doit aussi être repensé en fonction de l'enclavement/désenclavement des zones rurales. Là aussi, il faudra mesurer les risques d'un désenclavement de la région sur la vie des communautés locales. Une forte ouverture ne serait-elle pas une menace pour la «paisibilité» de la région, et une tentative de recomposition sociale et territoriale ne serait-elle pas en définitive une menace pour la durabilité ?

\subsection{L'allégement du travail de la femme et l'amélioration de l'accès de la population aux services de base (éducation, services sanitaires, etc.)}

L'enjeu majeur est de cerner la place des femmes comme acteur économique. La société rurale marocaine repose essentiellement sur le travail des femmes, or ces dernières ne sont que rarement valorisées, voire même reconnues comme acteur à part entière. L'un des objectifs de cette recherche est de souligner la place et le rôle des femmes dans la région, non dans une vision restrictive de type «genre et développement», mais avant tout dans une vision dynamique permettant un repositionnement clair des femmes en milieu rural. L'enclavement dont souffrent certaines localités et villages a largement joué dans cette marginalisation. Pourtant, les femmes ont un rôle important dans l'agriculture. Dans ce cadre, on évaluera les possibilités d'un accompagnement et d'une aide plus spécifiques.

\section{Références}

1. BEKEE, Expert Knowledge Modeling with Bayesian Belief Networks http://www.slideshare.net/jouffe/bekee

2. Ftaïta. Toufik, 2006, Anthropologie de l'irrigation. Les oasis de Tiznit (Maroc), Editions de l'Harmattan, 2006.

3. Ftaïta. Toufik, 2011, «Patrimoine hydraulique et préservation de la biodiversité en milieu aride, le cas des oasis marocaines», pp 185-206, In Dahou Tarik \& al (sous la direction), Pouvoirs, sociétés et nature au sud de la Méditerranée, Karthala.

4. Zakaria Kadiri \& al, «L'innovation institutionnelle dix ans plus tard : quelles opportunités pour les agriculteurs, et quels apprentissages pour les pouvoirs publics ? Le cas des Associations d'irrigants au Nord du Maroc», ISDA 2010, Montpellier 28-30 Juin 2010, http://hal.cirad.fr/docs/00/52/33/16/PDF/Kadiri_L_ innovation_institutionnelle.pdf 